\title{
Plans for first plasma operation of Wendelstein 7-X
}

\author{
T. Sunn Pedersen*, T. Andreeva, H.-S. Bosch, S. Bozhenkov, \\ F. Effenberg ${ }^{1}$, M. Endler, Y. Feng, D. A. Gates ${ }^{2}$, J. Geiger, \\ D. Hartmann, H. Hölbe, M. Jakubowski, R. König, H. P. Laqua, \\ S. Lazerson ${ }^{2}$, M. Otte, M. Preynas ${ }^{3}$, O. Schmitz ${ }^{1}$, T. Stange, \\ Y. Turkin and the W7-X Team \\ Max-Planck-Institut für Plasmaphysik, 17491 Greifswald, Germany \\ ${ }^{1}$ University of Wisconsin, Madison, WI, USA \\ ${ }^{2}$ Princeton Plasma Physics Laboratory, Princeton, NJ, USA \\ ${ }^{3}$ École Polytechnique Fédérale, Lausanne, Switzerland
}

30th September 2015

\begin{abstract}
Wendelstein 7-X (W7-X) is currently under commissioning in preparation for its initial plasma operation phase, operation phase 1.1 (OP1.1). This first phase serves primarily to provide an integral commissioning of all major systems needed for plasma operation, as well as systems, such as diagnostics, that need plasma operation to verify their foreseen functions. In OP1.1, W7-X will have a reduced set of in-vessel components. In particular five graphite limiter stripes replace the later foreseen divertor. This paper describes the expected machine capabilities in OP1.1, as well as a selection of physics topics that can be addressed in OP1.1, despite the simplified configuration and the reduced machine capabilities. Physics topics include verification and adjustment of the magnetic topology, testing of the foreseen plasma start-up scenarios and feed-forward control of plasma density and temperature evolution, as well as more advanced topics such as scrape-off layer (SOL) studies at short connection lengths, and transport studies. Plasma operation in OP1.1 will primarily be performed in helium, with a hydrogen plasma phase at the end.
\end{abstract}

\section{Keywords}

Stellarator; Wendelstein 7-X; Limiter; Operation; Planning

${ }^{*}$ Corresponding author, email: thomas.sunn.pedersen@ipp.mpg.de 


\section{Contents}

1 Introduction 2

2 Boundary conditions 3

2.1 Plasma facing components and energy per plasma pulse . . . . . . . . . . 3

2.2 Magnetic configuration . . . . . . . . . . . . . . . . . 4

2.3 Heating power . . . . . . . . . . . . . . . . . 5

2.4 Fueling and exhaust . . . . . . . . . . . . . . . . . 6

2.5 Available diagnostics . . . . . . . . . . . . . . . . 7

3 Flux surface measurements $\quad 9$

4 Wall conditioning and plasma startup 11

5 Expected pulse duration and stationarity of discharges 13

6 Limiter load distribution and edge physics $\quad 14$

$\begin{array}{llr}7 & \text { Plasma parameter control } & 17\end{array}$

8 Plasma current evolution and plasma current drive 18

9 Hydrogen operation $\quad 20$

10 Conclusions $\quad 21$

$\begin{array}{ll}\text { Acknowledgements } & 21\end{array}$

$\begin{array}{ll}\text { References } & 21\end{array}$

\section{Introduction}

The Wendelstein 7-X (W7-X) stellarator [1, 2, 3], presently under commissioning at the Max Planck Institute for Plasma Physics in Greifswald, is expected to start plasma operation in the second half of 2015. In order to allow for an early integral test of the main systems needed for plasma operation (magnet system, vacuum, plasma heating, control and data acquisition, etc.), the divertor units and most of the carbon tiles covering the wall protection elements [4] will not be installed for the first operational phase. Instead, following the five-fold symmetry of the device, five poloidal graphite limiters have been installed on the torus inboard side in those toroidal positions with the maximum vertical elongation of the magnetic surfaces ("bean-shaped planes") [5]. After approximately three months of initial operation (OP1.1), the un-cooled test divertor unit (TDU) [6] and the aforementioned graphite tiles will be installed. This installation is expected to take roughly one year, after which the next operation phase (OP1.2) will commence.

OP1.1 was introduced to allow for an accelerated, fully integrated commissioning of the main systems on W7-X, andan accelerated path to first plasma operation. This mitigates 
schedule risk, since many upgrades and improvements can be done in parallel with the TDU installation, including improvements whose necessity may only manifest themselves during first plasma operation.

One important element which concerns at the same time technique and physics is the measurement of the flux surfaces. Technically this verifies the success of the enormous engineering efforts in building the device, and scientifically, it is the first confirmation of the necessary topology of the magnetic field. In particular this includes the documentation of field errors and the tests how to reduce them. This can be done before plasma operation. In OP1.1 plasma operation, it is planned to investigate several interesting physics topics in preparation of the next operational phases. In section 2 , the boundary conditions for plasma operation in OP1.1 are described. In the following sections $3-9$, the planned physics program for OP1.1 is described, including numerical simulations of the scenarios that are foreseen. The conclusions follow in section 10. We note that the physics program is rather ambitious, and not all goals presented here may be achievable in OP1.1. It should be made clear already here that the majority of the physics objectives which have been defined for the W7-X project [7] will only be reached in later operational phases.

\section{Boundary conditions}

\subsection{Plasma facing components and energy per plasma pulse}

For OP1.1, many of the plasma-facing components are installed, but for the components that are designed to receive significant heat loads in later operation phases, the foreseen fine-grain graphite cladding is not installed, so that, for example, the $\mathrm{CuCrZr}$ cooling structures of the heat shields are uncovered. Most importantly, no divertor [8] will be installed in this first phase. For the absorption of convective plasma heat and particle fluxes in OP1.1, instead of a divertor, five graphite limiters are installed at the torus inboard side (see fig. 1). For the later operation phases, the convective plasma heat fluxes will be distributed over a much larger area provided by the divertor target plates, where pumping will also be implemented.

A maximum heating energy of $\sim 2 \mathrm{MJ}$ to be deposited in the plasma is therefore envisaged for each discharge. The limiters are designed to absorb this heat load, each laid out to absorb $400 \mathrm{~kJ}$ at heat fluxes up to $10 \mathrm{MW} / \mathrm{m}^{2}$ [5]. For a symmetric heat load distribution to the available limiter surface, the surface temperature will rise by $\sim 800 \mathrm{~K}$ in a $2 \mathrm{MJ}$ pulse. The limiters are not actively cooled, so a dwell time of several minutes between plasma pulses will be necessary, so that they can cool off, primarily through thermal radiation. The limiter shape is designed for uniform power distribution on the surface close to the last closed magnetic surface (LCMS), assuming an anomalous radial heat diffusivity of $3 \mathrm{~m}^{2} / \mathrm{s}$, and the limiter position and size is chosen such that none of the unprotected in-vessel structures is closer to the LCMS than 5-6 power decay lengths $[5]$. 


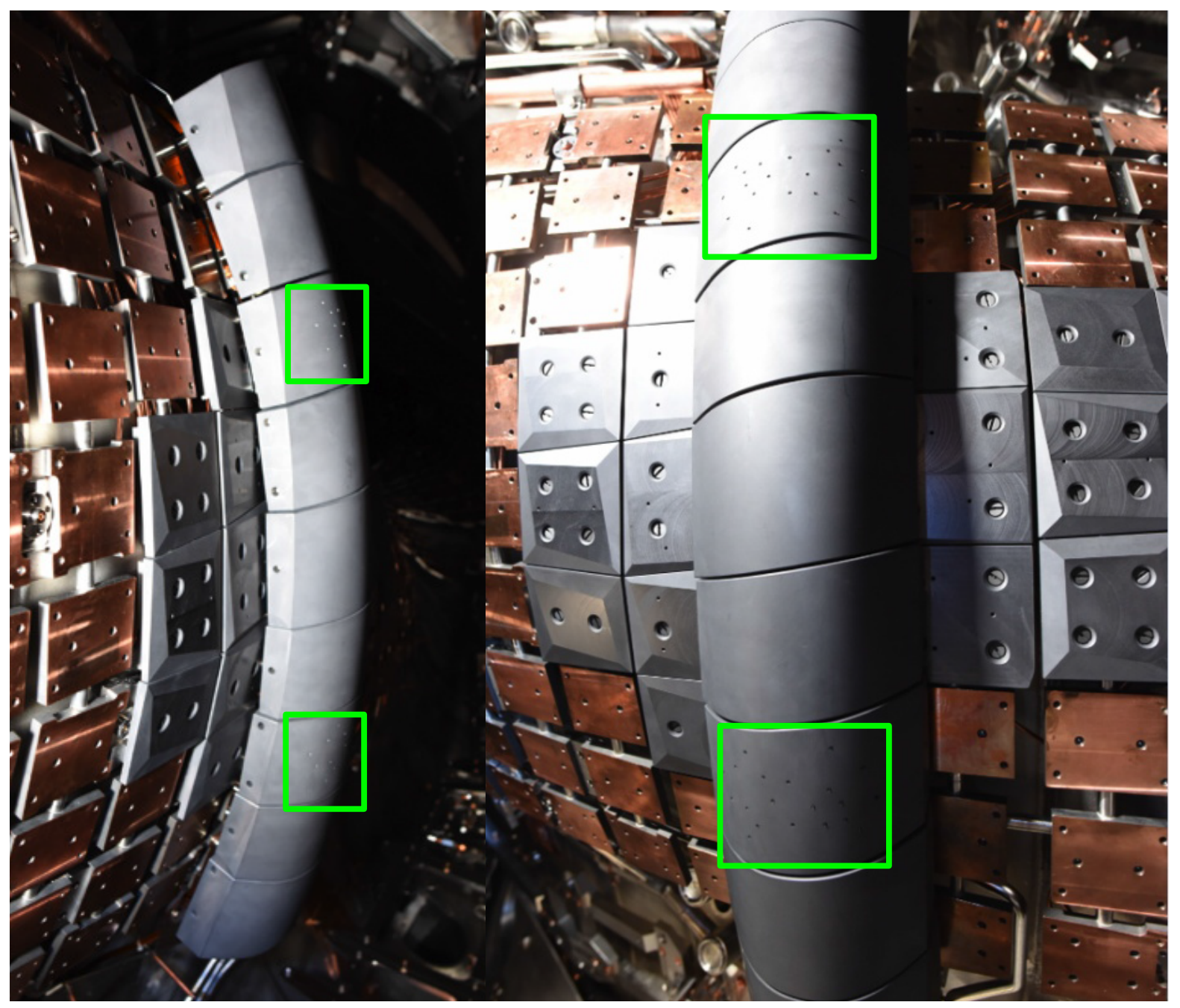

Figure 1: One of the five inboard limiters in the symmetry plane with bean-shaped plasma cross-section (left: toroidal view; right: radial view). In this limiter, several $0.9 \mathrm{~mm}$ diameter Langmuir probes are integrated in the third and in the seventh of the nine graphite tiles (visible inside the green frames, for the radial and vertical distribution see fig. 2).

\subsection{Magnetic configuration}

A special magnetic configuration was chosen for this limiter operation phase. It will have smooth, closed flux surfaces at the limiter surface as well as deep into the magnetic shadow of the limiters. This has been generated by reducing the rotational transform at the LCMS to $t \sim 0.87$, thus shifting the 5/5 magnetic islands of the W7-X "standard case" magnetic field configuration [9] far outward (see fig. 3). By eliminating edge islands, transport shortcuts to unprotected surfaces in the limiter shadow are avoided. The magnetic field strength on axis will be $B=2.5 \mathrm{~T}$, in order to ensure resonant second harmonic absorption of the microwave heating described in the following section. 


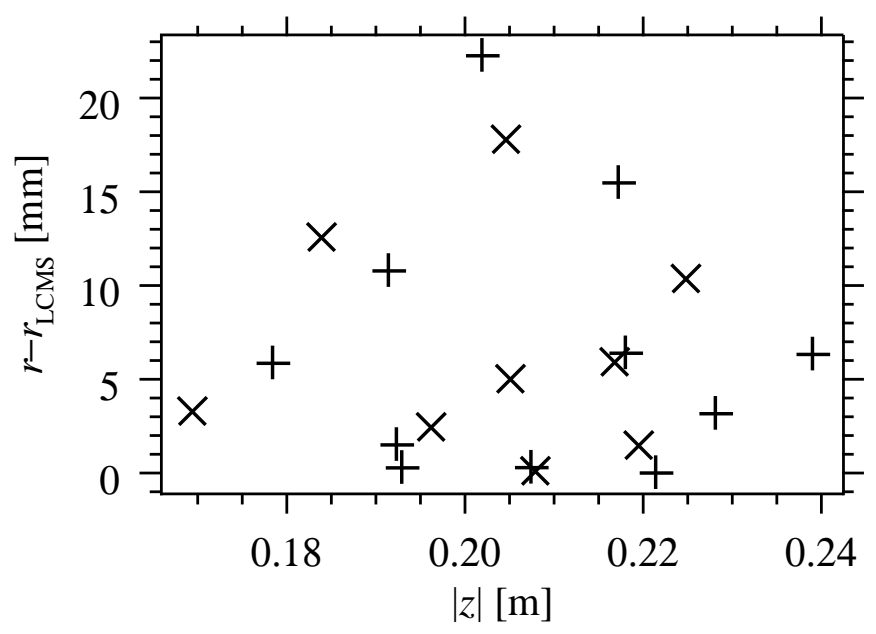

Figure 2: Spatial distribution of the Langmuir probe tips in the inboard limiter. The distance from the last closed magnetic surface $r-r_{L C M S}$ and the vertical position $z$ are indicated. The tips are arranged symmetrically in the two tiles indicated in fig. 1. The toroidal location on the two sides of the watershed is indicated by two different symbols.
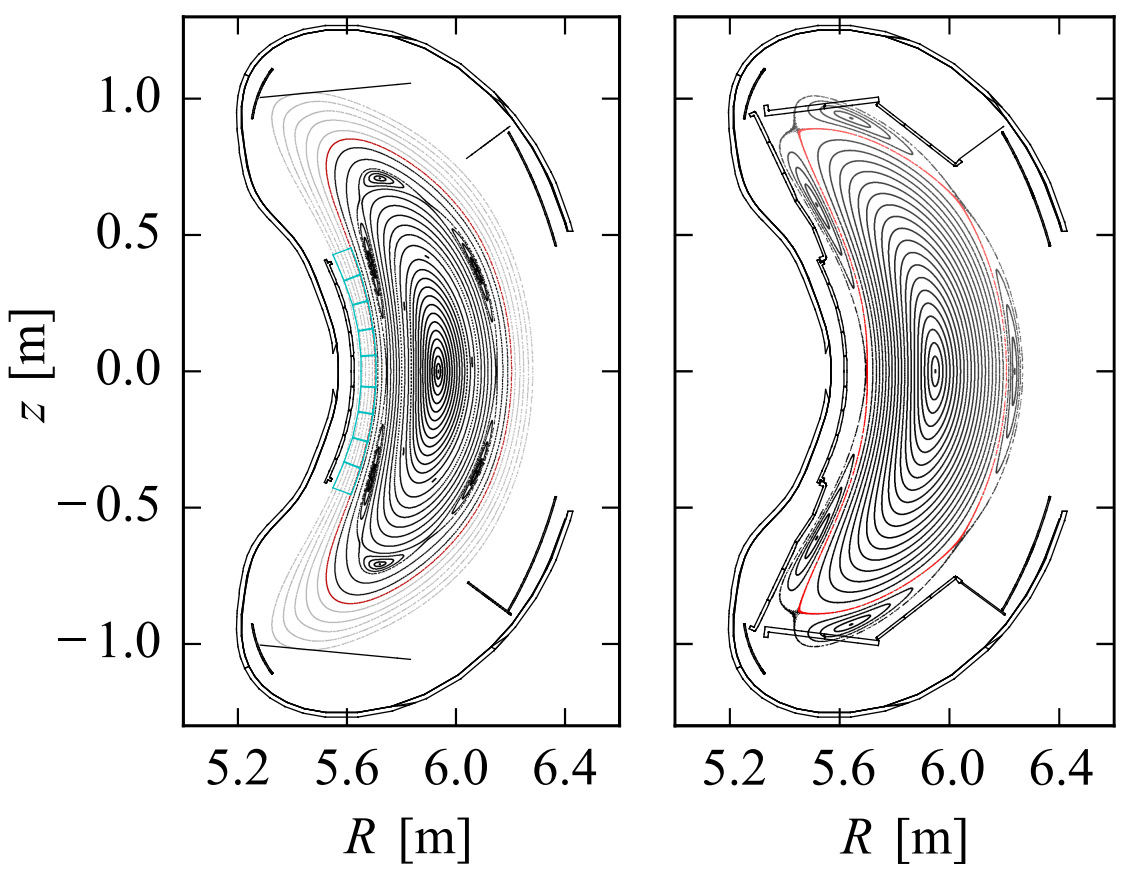

Figure 3: Poincaré plots of the flux surfaces in the bean-shaped symmetry plane for the OP1.1 limiter configuration (left) and for the "standard case" with the 5/5 island chain determining the last closed magnetic surface (LCMS, marked in red) (right). The limiter is shown in blue, the divertor frame (left) and the divertor and baffles (right) as well as the plasma vessel in black. In the left panel, the 5/6 island chain can be seen slightly inside the LCMS (marked in red). No large islands are visible in the limiter shadow (grey flux surfaces) up to the plasma vessel in the OP1.1 limiter configuration. The corresponding radial profiles of the rotational transform are shown in fig. 4.

\subsection{Heating power}

Six gyrotrons operating at $140 \mathrm{GHz}$ will be available to deliver an absorbed electron cyclotron resonance heating $(\mathrm{ECRH})$ power of $5 \mathrm{MW}$ in OP1.1. Applying the full available 


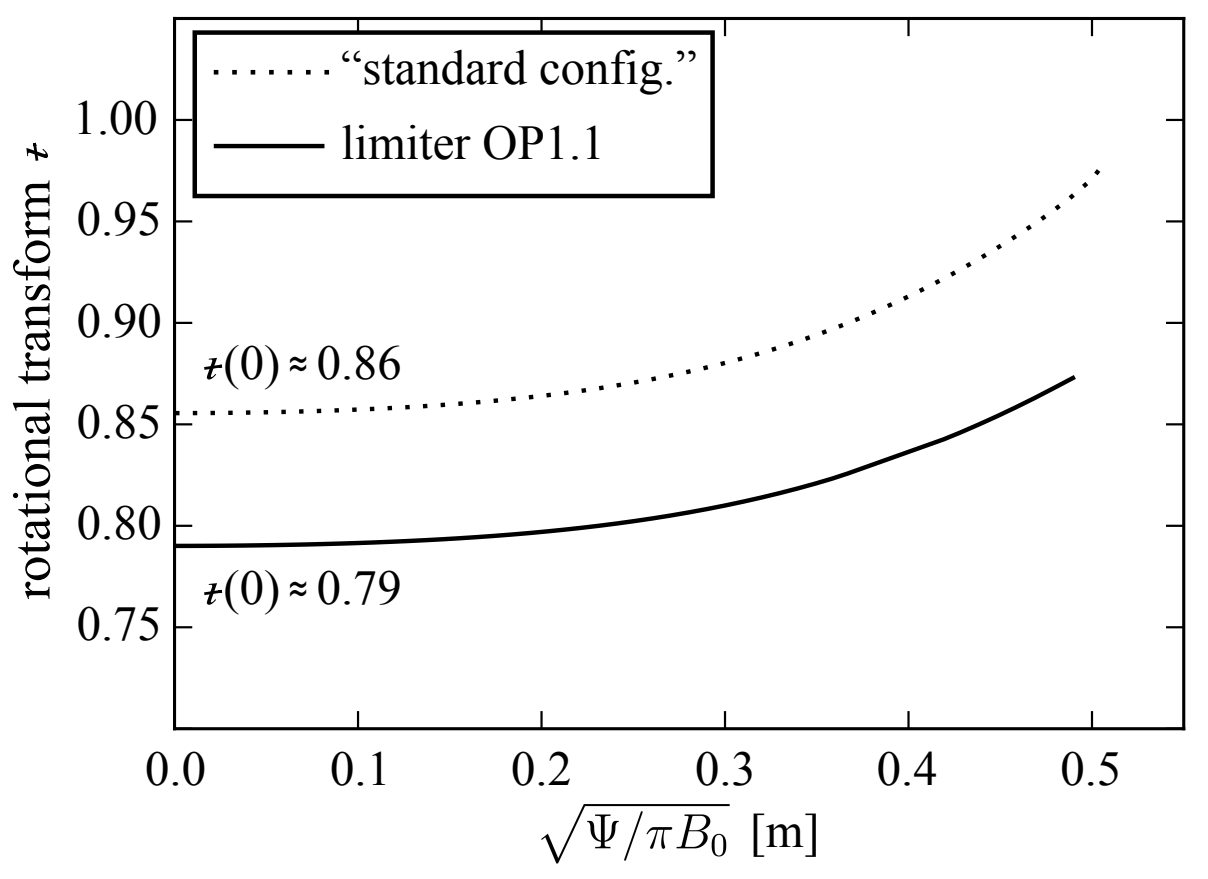

Figure 4: Radial profiles of the rotational transform for the two magnetic configurations shown in fig. 3.

power in OP1.1 with the $2 \mathrm{MJ}$ limit would restrict the discharge duration to $\tau \leq 0.4 \mathrm{~s}$ but of course longer pulses at lower heating power will be possible as well. Each of the six beam lines in use is equipped with a front steering mirror variable in toroidal and poloidal direction, allowing current drive experiments and on/off-axis heating, respectively. The Gaussian ECRH beams are focused towards the opposite wall of the plasma vessel with a beam waist of about $35 \mathrm{~mm}$ in the plasma center (around the major radius of the resonant plane) [10]. The power of each gyrotron can be modulated with a frequency up to $10 \mathrm{kHz}$, allowing power deposition experiments as well as transport studies. The other foreseen heating systems (NBI and ICRH) will only be available in later operation phases (starting in OP1.2, see section 1).

\section{$2.4 \quad$ Fueling and exhaust}

The W7-X plasma vacuum vessel will in OP1.1 plasma operation be pumped by 30 turbomolecular pumps [11], with an estimated total effective pumping speed of about $38 \mathrm{~m}^{3} / \mathrm{s}$ for hydrogen. This is the same system that will be used in OP1.2. Although this is adequate to reach the base pressure of $10^{-6} \mathrm{~Pa}$, and also predicted to be adequate for plasma exhaust and density control in OP1.2, this system will be less effective at controlling the plasma exhaust in OP1.1. The plasma exhaust will occur primarily at the five limiters, which are somewhat spatially separated from the future sub-divertor volume, where the pump ports are located. The central gas inlet system will have a relatively long characteristic time scale (on the order of $100-300 \mathrm{~ms}$ ). A faster gas inlet system is provided by two high-pressure gas boxes installed inside the plasma vessel about $20 \mathrm{~cm}$ from the last closed flux surface. Each of them can be operated independently with a non-inflammable, non-toxic gas (e.g., $\mathrm{He}, \mathrm{N}_{2}, \mathrm{Ne}$, Ar), or with a predetermined mixture of such gases. In 
future operation phases, these gas boxes will be part of the supersonic helium-beam diagnostics. These boxes are supplied with fast piezo-controlled valves capable of opening and closing in about $1 \mathrm{~ms}$, on the order of the time it takes the neutral gas to reach the plasma. Thus, pumping between plasma discharges will be swift, but it is not expected that the density can be held constant during OP1.1 discharges. Also, the density feedback control system, planned for future operation phases, is not expected to be operational in OP1.1. Thus, the density evolution may be steered to some degree by pre-programming of the gas inlet systems just mentioned, on a shot-to-shot basis, but the degree to which actual density control can be achieved remains open.

\subsection{Available diagnostics}

For OP1.1, the preparation of diagnostics proceeds according to their assigned priority. The diagnostics with highest priority are those that are needed for machine safety, or have physics missions in OP1.1 which are of central importance. Examples of the latter are given in sections 3-9. It is one of the goals of OP1.1 to commission as many diagnostics as possible, and to test their functionality during plasma operation. Here, we list the diagnostic systems expected to be ready in OP1.1, together with a short description of their role in OP1.1. A more detailed description with references can be found in [12]. We start the list with diagnostics required to assure the (safe) operation of the device and continue with diagnostics characterizing the plasma and discharge parameters.

- Two out of three manipulators of the magnetic flux surface mapping diagnostics are installed and will be used to verify the quality of the magnet system (see section 3 ).

- ECRH stray radiation detectors (sniffer probes) are installed to detect the level of such radiation. Their signals will be used to switch off the ECRH in case of insufficient absorption by the plasma. This is done in order to protect the invessel components from a high level of microwave stray radiation. In addition, 128 waveguide antennas embedded in the graphite tiles on the plasma vessel wall side opposite the ECRH launchers will serve as electron cyclotron absorption (ECA) diagnostic to monitor the non-absorbed ECRH power after a single pass of the plasma column.

- Although no significant neutron flux will occur in OP1.1, the neutron counters [13] are required for the license to operate W7-X. They were already calibrated with a neutron source in the plasma vessel.

- 10 video cameras with toroidal views will allow to fully monitor the interior of the plasma vessel [14]. Two of these views will be used by cameras with higher sensitivity to observe the fluorescent rods of the flux surface mapping diagnostic. One additional camera will be available in each of the four ECRH launchers monitoring those two limiters which are located opposite to the ECRH launchers and to detect possible arcing between the surrounding graphite tiles and their overheating by an ECRH beam, especially in case of wrong polarization settings [15]. 
- 10 immersion tubes with two cameras in the visible range and one near-infrared camera in each tube have been installed, which will later observe the divertor units and can in OP1.1 be used to monitor the complete number of limiter tiles, allowing the measurement of heat loads.

- In each limiter, two out of nine tiles were equipped with thermocouples, allowing temperature measurements of the $\mathrm{CuCrZr}$ holding structure, so that the shot-to-shot dwell time can be adjusted to avoid overheating of the holding structure.

- Two thermal He beams will be integrated into the divertor. For OP1.1, their gas inlets are attached to the divertor frames, a simplified observation system has been installed, and the beams will be operated with inert gases, in particular helium, neon, and nitrogen. These can also serve the operational purpose of fast gas inlets, as described in section 2.4.

- Five neutral pressure gauges of ASDEX type [16] have been installed for OP1.1.

- The line-integrated electron density will be measured by a single channel dispersion interferometer based on a $\mathrm{CO}_{2}$ laser [17]. Its beam is collinear with the laser beam of the Thomson scattering diagnostic (see below), which will allow direct comparison of the two independent density measurements.

- For electron temperature measurements with high spatial and temporal resolution, an electron cyclotron emission (ECE) diagnostic will be available with a 32-channel standard system and a 16-channel "zoom" system to increase the spatial resolution in a selected radial interval. In addition to the antenna observing from the low-field side, an antenna on the high-field side exists to provide information on suprathermal and current driven electrons [17].

- From Thomson scattering, 10 measurement points of electron temperature and density will be available in OP1.1 with their observation volumes in the outer half of the plasma cross-section (for the viewing geometry, see [18]). The 10 channels give $2 \mathrm{~cm}$ spatial resolution in the center and $3 \mathrm{~cm}$ resolution at the edge.

- Two bolometer cameras will allow a limited tomographic reconstruction of the twodimensional emission pattern in one of the triangular symmetry planes [19]. A number of additional channels is covered by Be filters of $10 \mu \mathrm{m}$ thickness for soft $\mathrm{X}$-ray $(>800 \mathrm{eV})$ detection.

- The magnetic equilibrium diagnotics (diamagnetic loops, continuous and segmented Rogowski coils and saddle coils) will be available to measure the diamagnetic energy, net toroidal plasma current and moments of the plasma current distribution (e.g., due to Pfirsch-Schlüter currents) [20].

- Two X-ray imaging spectrometers [21, 22, 23], a pulse height analysis system [24] and an extreme ultraviolet (XUV) overview spectrometer [25, 26] will allow to observe the emission of electrons and impurity ions in different locations with different 
spectral, temporal and spatial resolution and to obtain information on electron and impurity density, temperature and velocities.

- A single line-of-sight bremsstrahlung monitoring system will be operational in the spectral range $350-1000 \mathrm{~nm}$ to determine $Z_{\text {eff }}$.

- In one of the limiters, two tiles were equipped with a set of Langmuir probes (see figs. 1 and 2), which can be used to measure radial decay lengths of density and electron temperature within the SOL.

- A multi-purpose manipulator will be available at the outboard midplane [27]. Its head will be equipped with Langmuir probes and magnetic sensors. In later operating phases, different heads can be used, and an impurity gas inlet will be added.

- Two different reflectometer systems can be used to investigate density fluctuations, their spatial correlation, propagation velocity and wavenumbers [28].

\section{Flux surface measurements}

Two manipulators are installed in separate triangular cross-sections of the magnetic flux surfaces, each with an electron gun, and a rod covered with fluorescent ZnO:Zn powder (see fig. 5). The electron beam generated by one of the electron guns will follow the

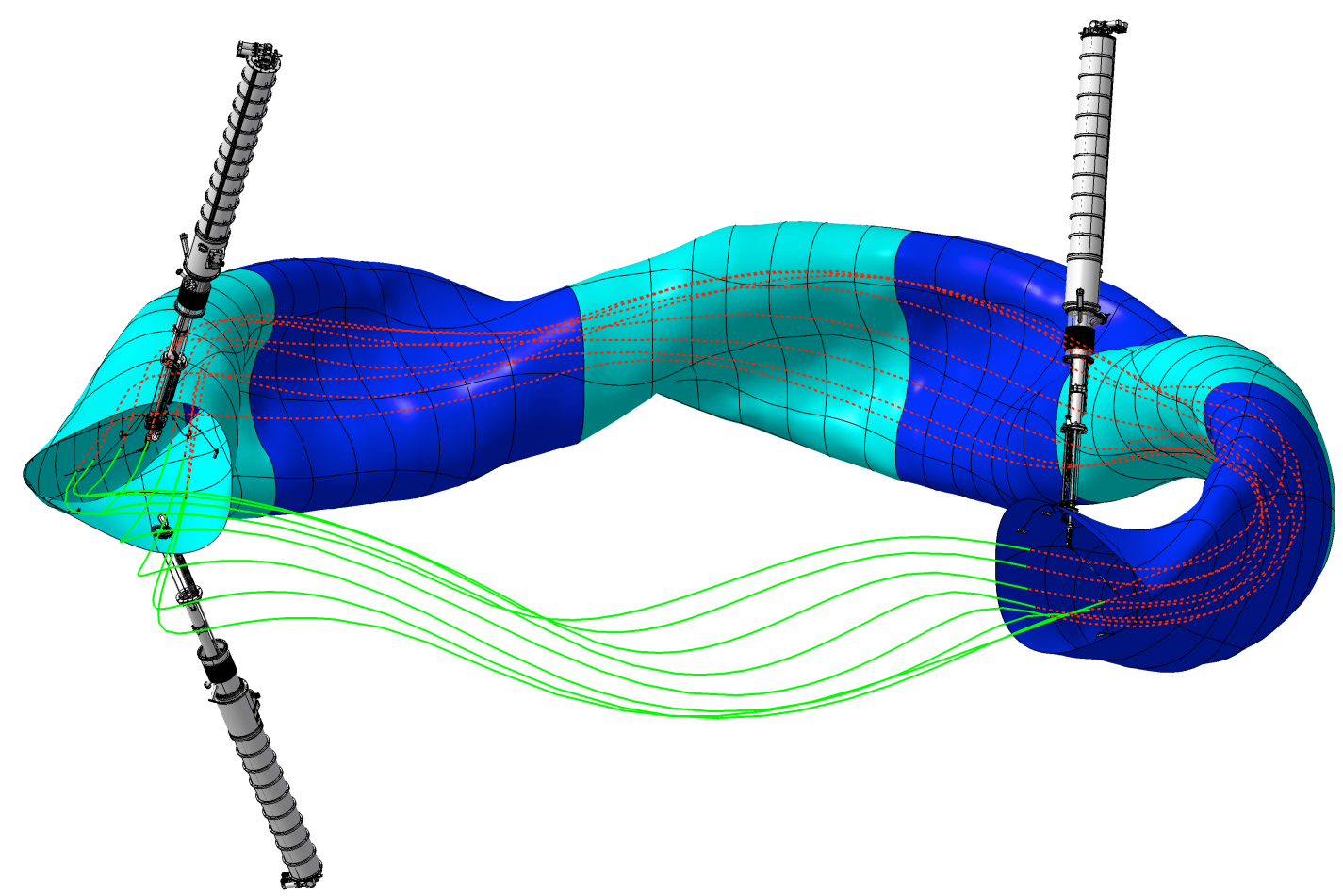

Figure 5: Manipulator positions of the W7-X flux surface mapping diagnostic together with the plasma vessel (in light and dark blue for the two flip-symmetric types of half module). The two upper manipulators are ready for operation, the lower manipulator will be added after OP1.1. 
magnetic field lines and hit the fluorescent rod in the other toroidal position. This rod will be swept through (almost) the entire cross-section of the plasma vessel, and the fluorescence from the electron beam hitting the rod will be recorded by a video camera. The outline of a magnetic flux surface is thus recorded. The technique is well known and has been used in various stellarators to map the magnetic flux surfaces, e. g. [29, 30, 31].

The first goal of the flux-surface mapping campaign in OP1.1 is to confirm that nested flux surfaces exist all the way out to the limiter, and that the expected value of the rotational transform was achieved, so that no large island chains exist near the last closed flux surface. A Poincaré plot of the standard limiter configuration is shown in fig. 3. A moderately sized internal island chain is visible at the $t=5 / 6$ surface, which should be clearly detectable in the flux surface mapping pictures. The location of the $t=5 / 6$ surface can thus be verified, which will ensure that no low order rational values can create islands in the near SOL behind the limiters. The convective plasma loads will then be effectively absorbed by the limiters, and only small convective heat loads will reach the thus shadowed plasma facing components, most of which are bare metal surfaces in OP1.1.

The second goal of the flux surface mapping campaign in OP1.1 will be to measure and eliminate resonant low-order magnetic field errors. The "standard case" magnetic configuration of W7-X has $t=1$ at the boundary and a natural resonant $n / m=5 / 5$ island chain at the edge (see fig. 3 ). This configuration is sensitive to $n=m$ components of a Fourier decomposition of the magnetic field error component perpendicular to the unperturbed flux surfaces, where $m$ and $n$ are the poloidal and toroidal mode numbers, respectively. The $n=m=1$, and, to a lesser degree, the $n=m=2$ error field components are particularly critical, since they change the topology of the $5 / 5$ island chain [32]. Although this would result in an asymmetric distribution of the heat load to the different divertor modules, it may prove difficult to detect it by flux surface mapping. Instead, the high- $t$ reference configuration which has $t$ just above 1 on the magnetic axis will be chosen, such that the axis itself will shift measurably for a resonant $1 / 1$ field error as low as $B_{11} / B_{0}=2 \cdot 10^{-5}$. In fig. 6 a comparison is shown between flux surfaces without error field, and with an error field with a $1 / 1$ component of $B_{11} / B_{0}=1 \cdot 10^{-4} \mathrm{~T}$. The error field in this simulation is generated by operating the five trim coils $[32,33]$ with different currents (following a sine function around the torus). The direction of the shift of the axis is uniquely related to the phase of the $1 / 1$ field error and the magnitude of the shift allows a direct measurement of the 1/1 field error amplitude (see fig. 7 ). The trim coils will then be used to eliminate the measured field errors - and to create well-defined error fields in order to provide accurate determination of $t$ on axis, and in general verify the principle used here [34].

Since the configuration used for this test has significant currents in the planar coils of the magnet system to obtain $t \sim 1$ close to the magnetic axis rather than at the plasma edge, the error field components might be slightly different from those of the "standard case". Nevertheless, this test is an important start in the investigation of the impact of error fields on the W7-X magnetic configurations. 

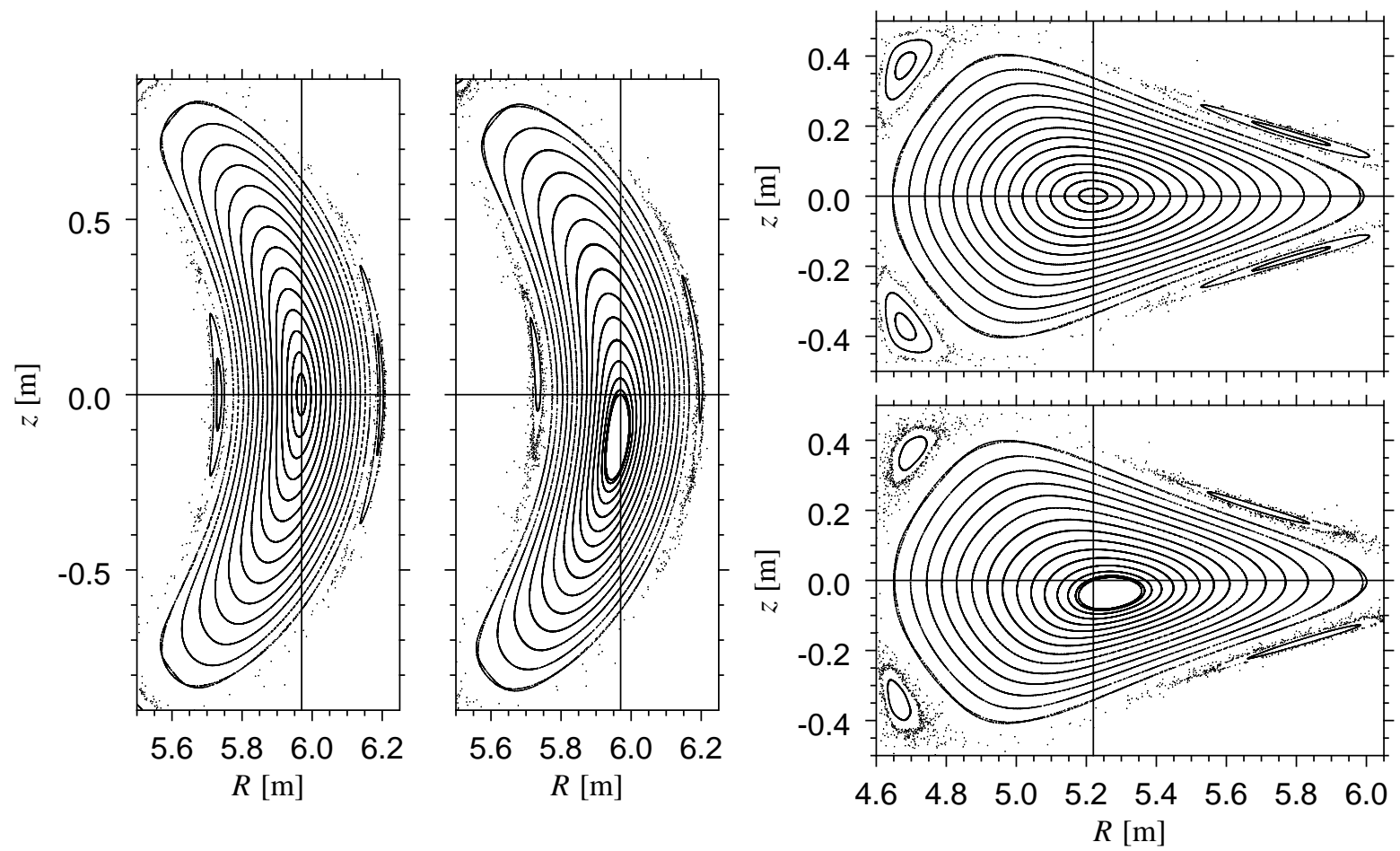

Figure 6: Poincaré plots showing the flux surfaces in the bean-shaped (left) and in the triangular plane (right) for $t=1.01$ on the magnetic axis without error field (left/top) and with an error field with a $1 / 1$ component of $B_{11} / B_{0}=10^{-4}$ (right/bottom). The shift of the plasma axis due to the error field (which was in this simulation generated by a certain set of currents in the trim and control coils) is clearly visible.

\section{$4 \quad$ Wall conditioning and plasma startup}

It is planned to bake the plasma vessel using hot water in the cooling/baking pipes under vacuum conditions to $150^{\circ} \mathrm{C}$ over a time period of 2 weeks. This should eliminate most of the water vapor. Several days of helium glow discharges will follow for further cleaning. The associated migration of helium into the lattice of metals and graphite is uncritical for OP1.1, because the first confined, ECRH generated plasmas will also be made from helium gas, and will also serve to condition the plasma-facing surfaces - primarily the limiters. The graphite limiters may need to be heated well above the $150^{\circ} \mathrm{C}$ to fully outgas their water inventory. This will be done by a series of short-pulse ECRH plasmas (e. g., with $500 \mathrm{~kW}$ for $100 \mathrm{~ms}$ ) during the first days of operation. The ratio of pulse length to pumping (non-pulse) time will have to be adjusted such that the limiter temperature is kept above $\sim 300^{\circ} \mathrm{C}$ for a duration of several hours. This limiter conditioning can be achieved simultaneously with the first efforts to extend the discharge length. It is expected that the pulse length and heating power can be increased significantly from the initial $50 \mathrm{~kJ}$ pulses to $2 \mathrm{MJ}$, over the course of just a few days. At first, the heating power will be increased and the discharge time will be kept short to minimize the accumulation of impurities released by the graphite tiles. In parallel to the scenario development, the plasma startup will be optimized regarding parameters such as the ECRH heating power, the neutral gas pressure as well as other parameters like on/off-axis heating, in order 


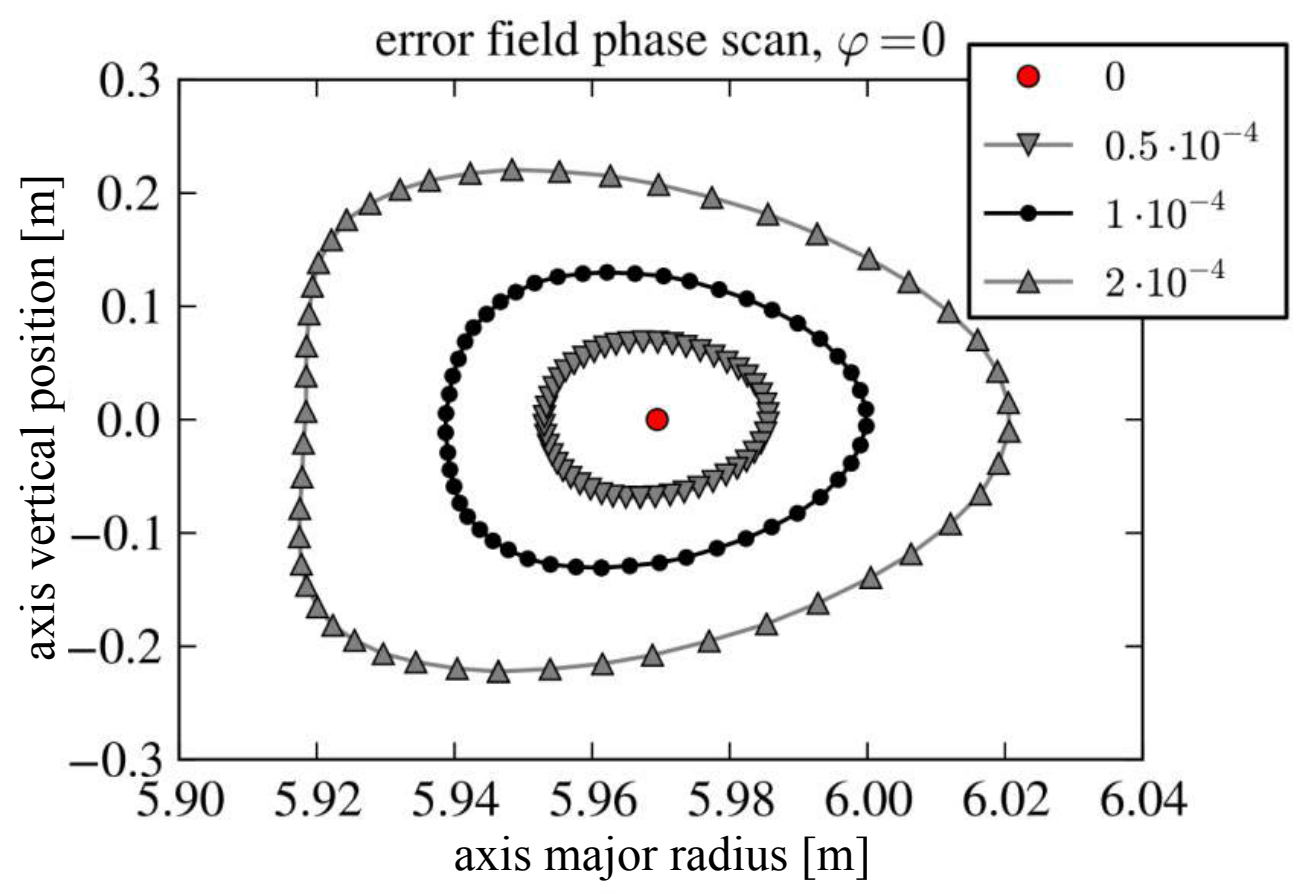

Figure 7: Location of the magnetic axis in the bean-shaped plane $(\varphi=0)$ for different amplitudes and phases of a magnetic error field consisting mainly of a $B_{11}$ component. The shifts are shown for three different amplitudes $B_{11} / B_{0}$ of the error field, as indicated by the different symbols.

to minimize the non-absorbed power during this early phase of the plasma discharge. Furthermore, ECRH power modulation will allow to determine the absorption position.

The pulse extension development and other physics tasks will be with helium plasmas first. It is known from previous fusion experiments that the walls can absorb and subsequently release significant amounts of hydrogen when hydrogen plasmas are used. Without a divertor, there is a risk that the shot-to-shot reproducibility of the plasma density evolution will be poor in this early campaign. Helium, being a noble gas, is much less likely to be loaded to the walls than hydrogen, and the shot-to-shot reproducibility of helium plasma density evolution is expected to be better.

A further observation favouring He for the first plasma operation campaign is the shorter delay between application of ECRH power and plasma startup in He as compared with $\mathrm{H}$ (for related experiments in the WEGA stellarator, see [35]).

After achieving a maximum pulse time of several seconds, requiring a stable feed forward scenario, further ECRH power modulation experiments may allow the first transport studies, even though steady-state conditions will not be reached (see section 5). 


\section{Expected pulse duration and stationarity of dis- charges}

At the available $5 \mathrm{MW}$ of heating power, the $2 \mathrm{MJ}$ pulse discharge limit implies a discharge time of up to $400 \mathrm{~ms}$, whereas discharges of up to $2 \mathrm{~s}$ could be operated with $1 \mathrm{MW}$ of heating power. To simulate the evolution of temperature, radial electric field and current density profiles, we use a transport model with a fixed density profile, neoclassical transport coefficients from the DKES code [36] and a prescribed radial profile of anomalous transport coefficients [37] ( $\chi_{\mathrm{e}}$, ano is small in the confinement region and increasing up to $2 \mathrm{~m} / \mathrm{s}^{2}$ at the plasma boundary). The ECR deposition profile is calculated by the TRAVIS code [38]. In fig. 8, we show the resulting profiles of temperature and radial electric field

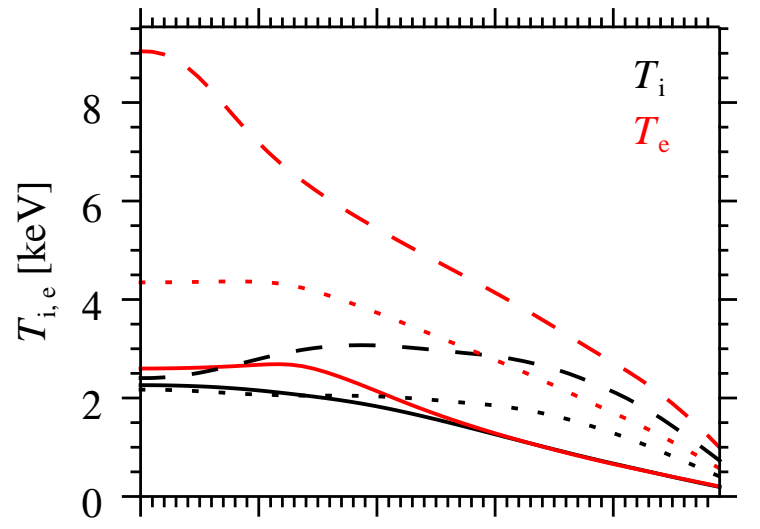

$\begin{array}{lllll}0.0 & 0.1 & 0.2 & 0.3 & 0.4\end{array}$ $r[\mathrm{~m}]$

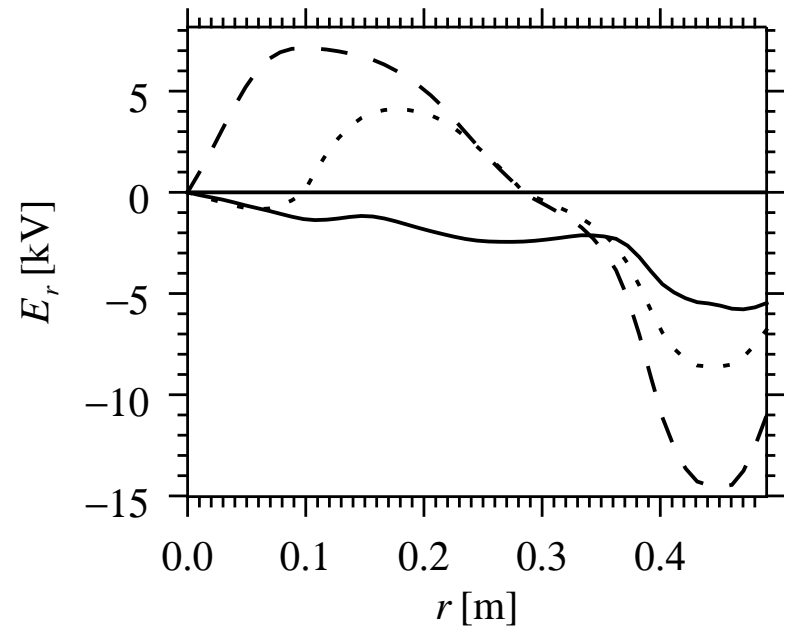

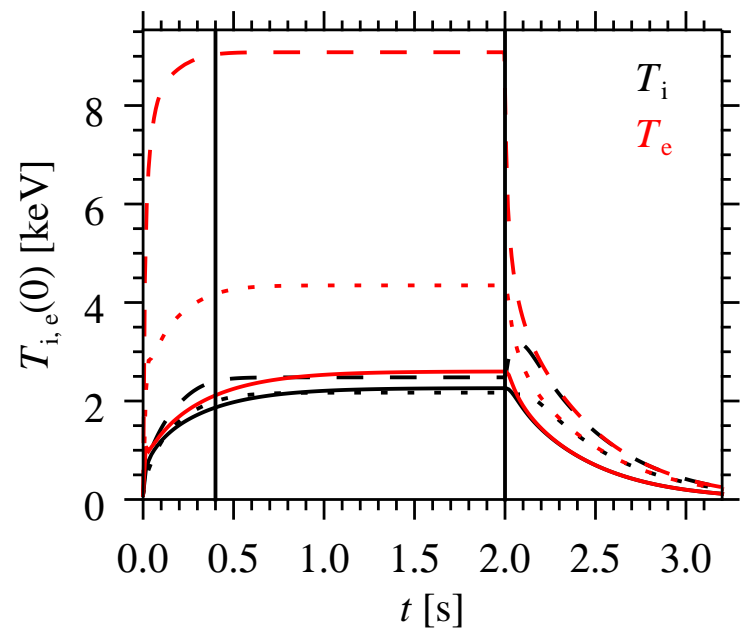

- $n_{\mathrm{e}}(0)=2 \cdot 10^{19} \mathrm{~m}^{-3}, P=1 \mathrm{MW}$, radial profiles at $t=2 \mathrm{~s}$

$-n_{\mathrm{e}}(0)=5 \cdot 10^{19} \mathrm{~m}^{-3}, P=1 \mathrm{MW}$, radial profiles at $t=2 \mathrm{~s}$

- $n_{\mathrm{e}}(0)=5 \cdot 10^{19} \mathrm{~m}^{-3}, P=5 \mathrm{MW}$, radial profiles at $t=400 \mathrm{~ms}$

Figure 8: Radial profiles of ion and electron temperature and of the radial electric field (left) from the transport simulation cases $A$ (dotted lines), $B$ (solid lines) and $C$ (dashed lines) at the end of the discharge, and time evolution of the central temperatures. (The evolution for case $C$ was computed for $2 \mathrm{~s}$, although the discharge length will be limited to $400 \mathrm{~ms}$ at a heating power of $5 \mathrm{MW}$.) The simulations were performed for discharges in He.

for a central electron density of $2 \cdot 10^{19} \mathrm{~m}^{-3}$ and $1 \mathrm{MW}$ heating power (case A) and for a central electron density of $5 \cdot 10^{19} \mathrm{~m}^{-3}$ and $1 \mathrm{MW}$ (case B) or $5 \mathrm{MW}$ (case C) heating 
power, as well as the time evolution of the central temperatures. After $2 \mathrm{~s}$ (cases A and B) or $400 \mathrm{~ms}$ (case C), the temperatures (and temperature profiles) are almost stationary. The energy confinement times in these simulations are $330 \mathrm{~ms}$ (case A), $470 \mathrm{~ms}$ (case B) and $260 \mathrm{~ms}$ (case C), which compares with values $\tau_{\mathrm{E}}^{\mathrm{ISS} 04}$ of the International Stellarator Scaling of 2004 (ISS04 [39]) of $200 \mathrm{~ms}$ (case A), $330 \mathrm{~ms}$ (case B) and $120 \mathrm{~ms}$ (case C). The expected particle confinement time is on the order of $700 \mathrm{~ms}$.

In contrast, the expected $L / R$ time scales at high performance are much longer than the discharge length in OP1.1: A rule of thumb for the $L / R$ time of the W7-X plasma is $\tau_{L / R} \sim 2 \mathrm{~s} \cdot\left(T_{\mathrm{e}}(0) / 1 \mathrm{keV}\right)^{3 / 2}$ (where the coefficient of $2 \mathrm{~s}$ may vary by a factor of $2-$ 3 , depending on the fraction of trapped particles and on the the radial profile of the electron temperature). Central electron temperatures at $5 \mathrm{MW}$ of heating are expected to be around $9 \mathrm{keV}$ (see fig. 8), so $\tau_{L / R} \sim 50$ seconds. At lower heating power of $1-2 \mathrm{MW}$, $T_{\mathrm{e}}(0) \sim 2-5 \mathrm{keV}$ is expected, and the discharge duration could be $1-2$ seconds. The corresponding $L / R$ time is, however, still clearly above the length of the discharge. At still lower central electron temperatures, it will probably be difficult to maintain a stable discharge at a reasonable density.

To summarize, discharges in OP1.1 will not achieve stationary toroidal current densities and may not achieve stationary pressure profiles. This should not come as a surprise - it just underlines the point that a proper exhaust concept is of central importance but this must be kept in mind when planning discharge scenarios, and when analyzing data of OP1.1.

\section{$6 \quad$ Limiter load distribution and edge physics}

The limiter plasmas provide a unique opportunity to study the recycling, refueling and sputtering processes with a short geometrical distance between the targets and the plasma core (see [40] and section 9). Although configurations without edge islands intersected by the divertor targets will be possible later, the divertor targets have a larger distance to the plasma core than the limiters do (see fig. 3). Due to shadowing effects from one limiter onto another, the five toroidally localized limiters generate a complex pattern of connection lengths: Each limiter will have two regions with short connection lengths (about $36 \mathrm{~m}$ and $43 \mathrm{~m}$ ), and one with a longer connection length (about $80 \mathrm{~m}$ ) (see figure 9). The open field line bundles with different connection lengths form a helical scrape-off layer and cause an anisotropic plasma boundary structure for the startup plasma [41]. If the anomalous transport perpendicular to the magnetic field is simulated by a Monte-Carlo test particle diffusion model [42], the pattern of different connection lengths on the limiter is reflected by the heat load (see fig. 9). The pattern can be shifted vertically by small variations in $t$. In OP1.1, the limiters will be observed by infrared cameras, allowing measurements of the heat load patterns. Two tiles of one limiter are also equipped with a number of Langmuir probes, which will provide complementary data on the radial density and temperature profiles in the SOL.

Before detailed investigations of the heat load distribution on individual limiters, measurements and corrections of asymmetric heat loads on the five limiters will be performed. These asymmetries could be caused by either the as-installed uncertainty in the limiter 

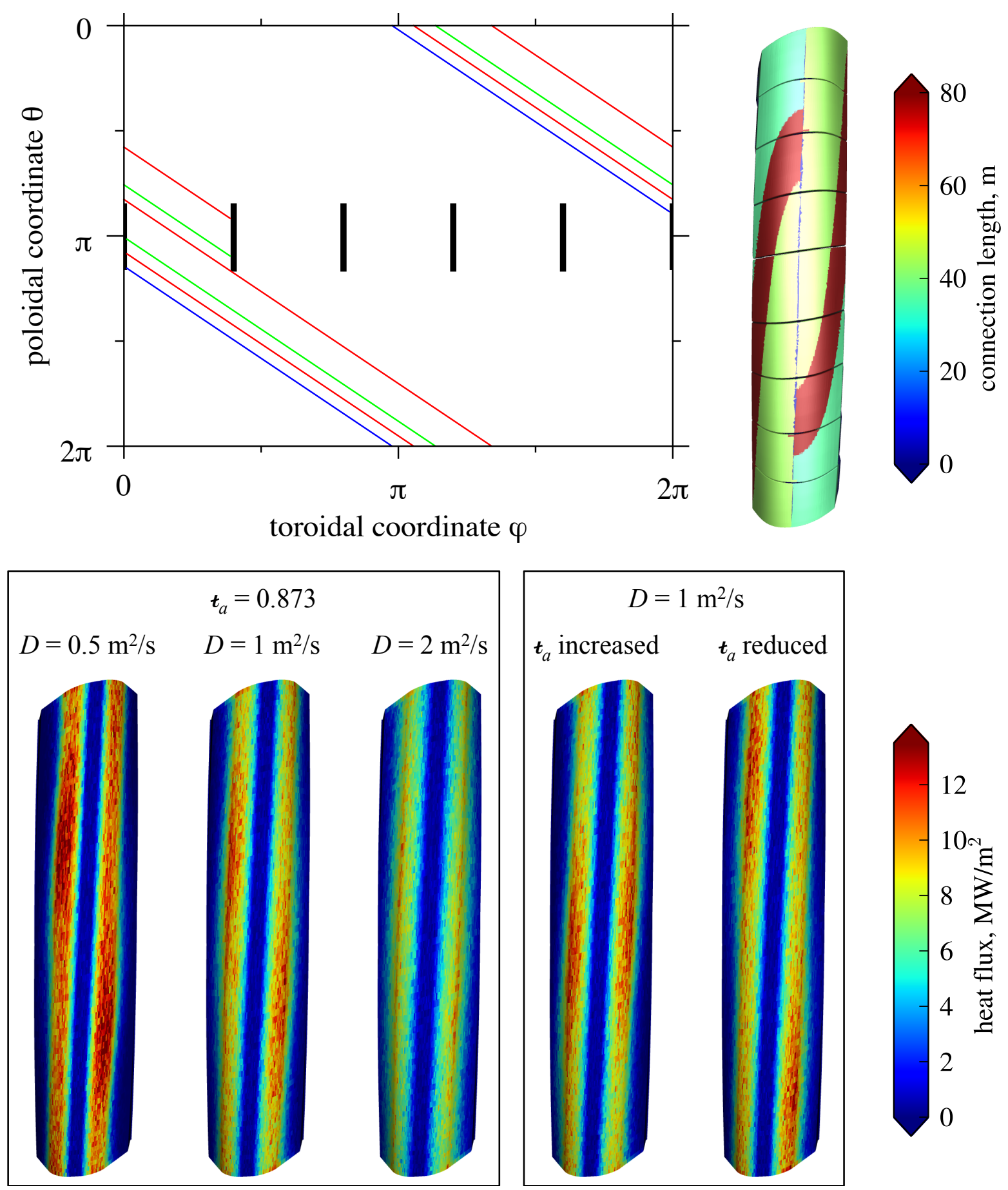

Figure 9: Connection lengths in the limiter shadow: on top, the case of the limiter standard configuration with $t_{a}=0.873$ is shown. Three types of field lines are indicated in the winding off in magnetic coordinates (with $\theta=0$ in the outboard midplane). The colours roughly correspond to the colour code for the connection lengths on the surface of a limiter, shown on top right (from [5]). The resulting power loads, calculated using a field line diffusion model, are depicted at the bottom (from [5]), with three different values for the diffusion coefficient in the limiter standard configuration (bottom left). The higher loading in the regions of longer connection length is clearly visible. In addition, the effect of a slight variation of the edge rotational transform $t$ on the location of the higher loaded regions is shown (bottom right). 
locations, on the order of $1.5 \mathrm{~mm}$, or from magnetic field errors. Numerical simulations show that the expected asymmetries from these sources can be balanced by shifting the flux surfaces in major radial direction by use of the trim coils. Conversely, asymmetric heat loads could be induced by the trim coils, to verify their action.

A measurement of the scrape-off layer width in the limiter configuration will contribute to the comparison with the much larger connection lengths $L_{\mathrm{c}}$ along field lines between the upstream stagnation point and the target plates in island divertor configurations. Many SOL models predict that the width of the power-carrying layer $\lambda_{q}$ scales linearly with $L_{\mathrm{c}}$ (e.g. [43]). Indeed this is the generic result one would expect if the perpendicular transport rate is independent of the connection length. The more detailed analysis in [40] also takes into account the recycling of neutrals and impurity transport.

Here, we use the 3D plasma fluid and kinetic neutral transport code EMC3-Eirene to study limiter heat loads and the effects of carbon impurities sputtered from the limiter surfaces and of actively seeded nitrogen for different values of the density at the LCMS. A heating power of $4 \mathrm{MW}$ in a hydrogen plasma, an anomalous diffusion of $D_{\perp}=1 \mathrm{~m}^{2} / \mathrm{s}$ and $\chi_{\perp}=3 D_{\perp}$ are assumed for these simulations. The resulting heat load deposition patterns are shown in fig. 10, neglecting (a) and taking into account (b) radiative losses from

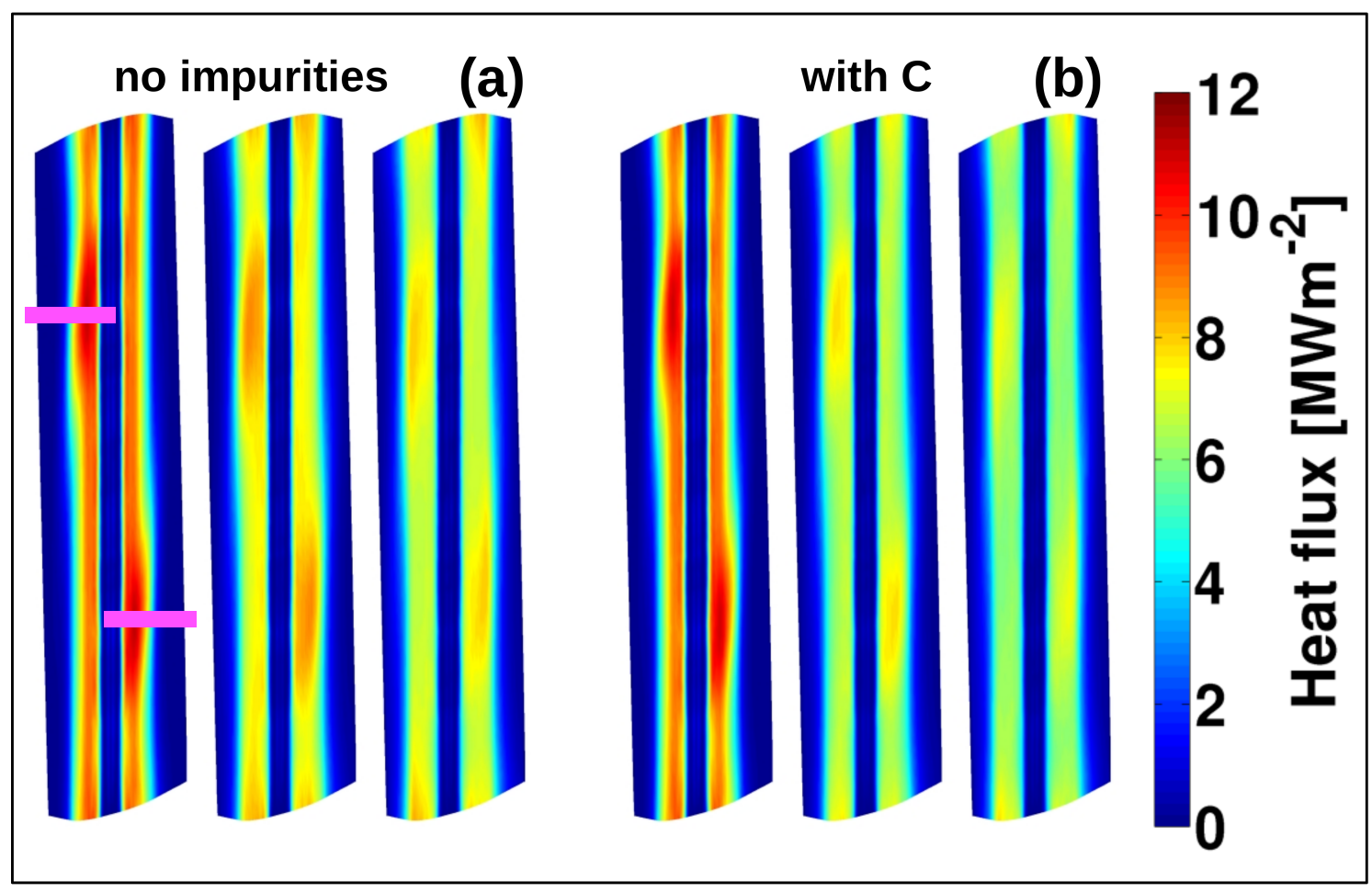

Figure 10: Limiter heat load deposition patterns for (a) three density scenarios (from left to right: $n_{L C M S}=0.1,0.9,1.7 \cdot 10^{19} \mathrm{~m}^{-3}$ ) with a heating power of $4 \mathrm{MW}$, and (b) the same three density scenarios as in (a), but including the effect of sputtered carbon with a chemical sputtering yield of $Y_{\text {chem. }}=0.03$. The peak heat loads shown in fig. 11 are determined in the positions marked by the magenta lines in the leftmost frame.

sputtered carbon as impurity in the plasma. The same pattern as in the simpler model (fig. 9) is reproduced. Increasing the density in the simulation results in a decrease of 
the peak load from $P_{\text {depo, } \max } \approx 11.8 \mathrm{MW} / \mathrm{m}^{2}$ down to $P_{\text {depo, } \max } \approx 8.7 \mathrm{MW} / \mathrm{m}^{2}$. For the simulation cases including carbon radiation, a chemical sputtering yield of $Y_{\text {chem. }}=0.03$ was assumed. In the lowest density case the intrinsic impurities have no significant impact on the peak load. However, in the higher density cases, the peak load is reduced by about $10 \%$ due to the intrinsic impurity radiation, relative to the corresponding cases without carbon (see fig. 11). Therefore, the feasibility of controlled heat flux mitigation by addi-

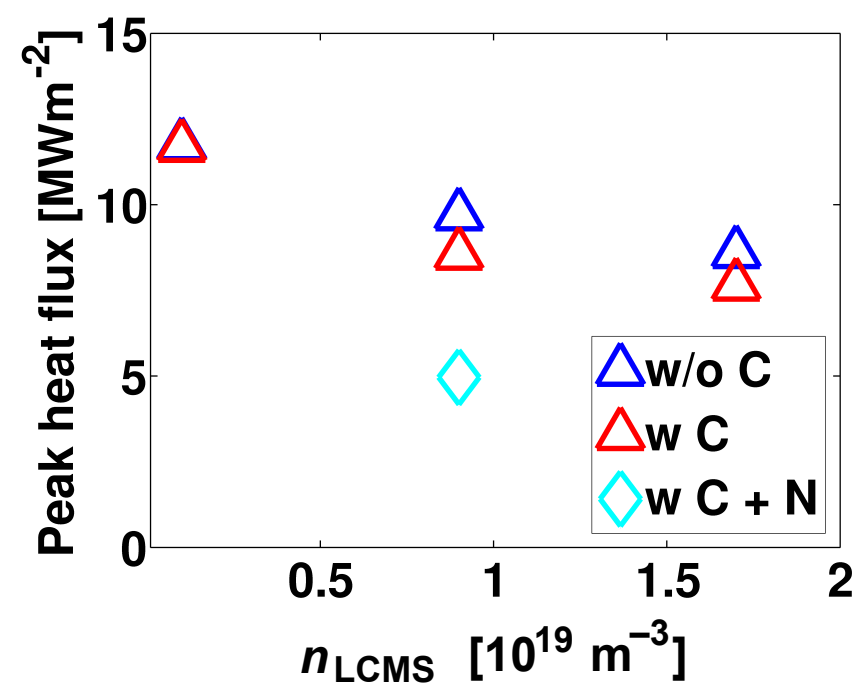

Figure 11: Limiter peak heat loads from EMC3-Eirene simulations for $4 \mathrm{MW}$ heating power in hydrogen plasmas: without impurities (dark blue triangles), including the effect of sputtered carbon (red triangles), and with the combined cooling effect of sputtered carbon and seeded nitrogen (light blue diamond, only one density case).

tional nitrogen seeding is demonstrated for the density case with $n_{\mathrm{LCMS}}=0.9 \cdot 10^{19} \mathrm{~m}^{-3}$ : Assuming a local nitrogen influx of $I_{\mathrm{N}}=5.2 \cdot 10^{21} \mathrm{~s}^{-1}$, the total radiative losses increase to $40 \%$, causing a peak load reduction down to $P_{\text {depo, } \max } \approx 4.9 \mathrm{MW} / \mathrm{m}^{2}$. In fig. 12 , a comparison of the limiter heat load deposition patterns is shown between pure hydrogen, sputtered carbon and the combination of sputtered carbon and injected nitrogen. The values of the maximum peak heat loads are compared in fig. 11 for all cases discussed. The location of the maximum load on the limiter is indicated by the magenta lines in the leftmost frame of fig. 10. These results are achieved in a first approach, assuming constant anomalous transport coefficients within the SOL and also in the confinement domain near the LCMS. Core radiation from heavier impurities is ignored. Such radiation could terminate a limiter discharge at lower values of $n_{\text {LCMS }}$ than assumed in this study. Further studies taking into account different transport conditions in the core are ongoing.

\section{Plasma parameter control}

ECRH will be the only heating method in OP1.1. The electron temperature is expected to be rather well controlled, on a shot to shot basis, by adjusting the ECRH power. The ion temperatures will, in addition to the ECRH input power, depend on the pulse length and plasma density, as it takes time for the electron population to transfer its energy to the 


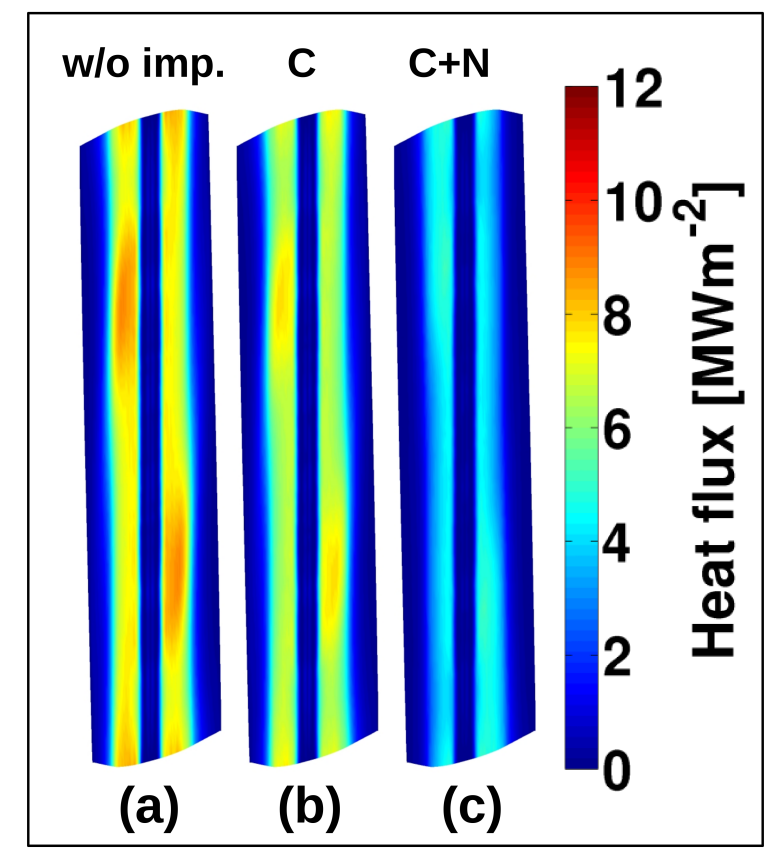

Figure 12: Limiter heat load deposition patterns for $n_{L C M S}=0.9 \cdot 10^{19} \mathrm{~m}^{-3}$ with a heating power of $4 \mathrm{MW}$. (a) pure hydrogen, (b) including the effect of sputtered carbon with a chemical sputtering yield of $Y_{\text {chem. }}=0.03$, (c) additional nitrogen seeding witn an influx of $I_{N}=5.2 \cdot 10^{21} \mathrm{~s}^{-1}$ ((a) and (b) correspond to the middle density cases of fig. 10).

ions through collisions. The fast actuators for discharge control in OP1.1 will be the preprogrammed control of the ECRH, allowing on- and off-axis heating with complex time evolution of the heating profiles, as well as the high-pressure fast piezo-valve gas boxes that will later be used for the helium beam diagnostic and active divertor gas fueling. Both of these systems can be controlled on time scales below $10 \mathrm{~ms}$, and will be used in a feed-forward sense. Feedback loops for density and temperature control are not expected to be yet operational in OP1.1.

\section{Plasma current evolution and plasma current drive}

For first tests of the electron cyclotron current drive (ECCD) and of the Rogowski coils measuring the total toroidal plasma current, we plan discharges which differ in their toroidal plasma current. As discussed in section 5, the toroidal plasma current and its radial density distribution will be far from stationary in OP1.1 discharges. However, measurable differences in driven toroidal current between different discharges should be achievable. This will be done by using ECCD either parallel or antiparallel to the direction of the bootstrap current, and by choosing the heating power, current drive power, and pulse length appropriately. This choice is nontrivial given the boundary conditions of OP1.1, as explained in the following. A longer discharge at lower ECRH power implies a lower temperature so that the $L / R$ time is relatively short, and a net current would appear. On the other hand, it also implies a less efficient current drive, and less ECRH power available to drive the current. Vice versa, a stronger ECRH would give higher 
temperatures, which increases the current drive efficiency, but also limits the pulse length and increases the $L / R$ time of the plasma. Although unable to claim we have found the optimum trade-off, we present here a modeled scenario where a clearly measurable driven toroidal current should be achievable in OP1.1.

We use the transport model described in section 5 and show in fig. 13 the resulting
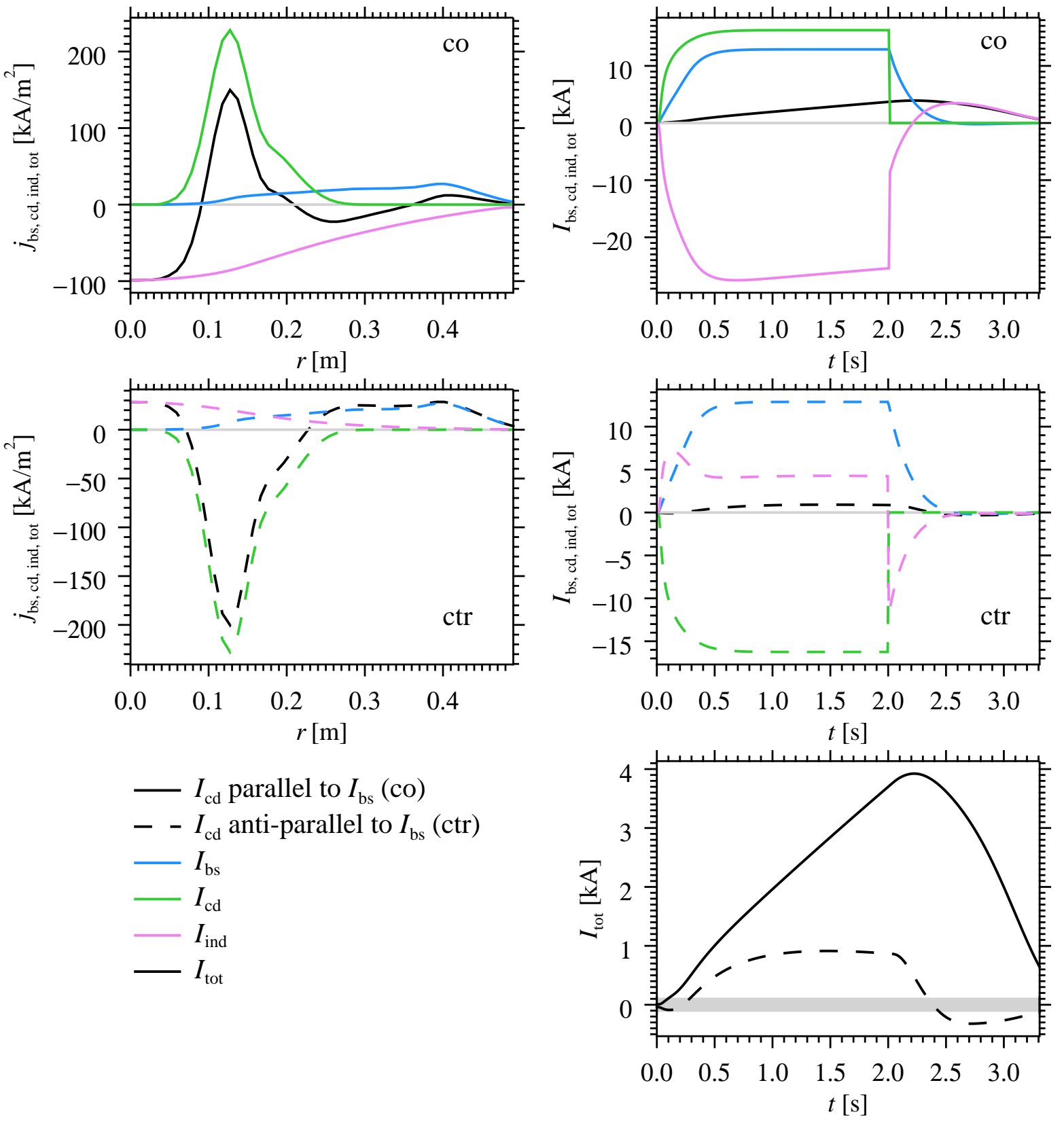

Figure 13: Transport simulation for discharges in He with 1 MW ECCD parallel (top row) and anti-parallel (middle row) to the bootstrap current. Radial profiles of the toroidal current densities are shown for the moment right before switching off the ECR power at $2 \mathrm{~s}$ (left column), the time evolution of these contributions to the net toroidal current is depicted in the right column. The evolution of the total current for the co and counter cases is shown enlarged in the bottom right panel, together with the uncertainty of the Rogowski coil measurement as a grey band around 0. 
radial current density profiles in a discharge in He with $1 \mathrm{MW}$ ECRH after $2 \mathrm{~s}$ together with the evolution of the total toroidal plasma current during and after the discharge. These are the same conditions as for case A of section 5, but with the ECRH launcher set to drive a toroidal current. Due to the $L / R$ time of $\sim 13 \mathrm{~s}$, the toroidal current densities are still far from equilibrated, and most of the bootstrap and ECR driven currents (blue and green in fig. 13) are masked by the induced self-shielding currents (violet in fig. 13). Therefore, the total toroidal current at the end of the discharge differs by only $\sim 2.5 \mathrm{kA}$ for the co- and counter current drive cases. This is, however, well above the expected measurement accuracy of the W7-X Rogowski coils, which have been designed to measure toroidal plasma currents down to as low as $0.1 \mathrm{kA}[20]$.

\section{$9 \quad$ Hydrogen operation}

Towards the end of OP1.1, $\mathrm{H}$ discharges will be performed in preparation of the next operation phase, and for a comparison of the discharge behavior between He and H. After the formation of a robust scenario for cleaning the graphite limiter in case of uncontrolled plasma density increase and subsequent radiative collapse, the hydrogen plasma program will evolve along the same lines as the He-plasma program sketched out above, but given the limited amount of time, it may not be possible to repeat all experiments that were performed in He. Priority will be given to startup plasma optimization and commissioning of spectroscopic diagnostics that require hydrogen operation, as well as to any particularly interesting or surprising findings from the He operation phase. Hydrogen operation will also allow a comparison with edge transport modelling [40], and a more direct comparison with discharges in divertor configurations in later operating phases, which will be performed mostly with hydrogen, and eventually, deuterium.

Likewise, hydrogen operation will allow the comparison with W7-AS results, since W7AS was operated with a set of 10 inboard limiters for some time before the installation of the divertor [44]. As the distance between limiter surface and plasma center is much smaller than between divertor targets and plasma center, recycling neutrals can penetrate deeper into the plasma, leading to stronger central refuelling, in particular at low density. If radial density profiles are measured in density and heating power scans, indications of the thermodiffusion predicted for central ECRH depostion may be found [45]. Thus, hollow density profiles would confirm the need for pellet injection in later high density operation.

In low density hydrogen operation at high heating power, charge exchange neutrals could reach plasma facing components in the limiter shadow. This might lead to increased influx of $\mathrm{Fe}$ and $\mathrm{Cu}$ due to sputtering. The careful investigation of such discharges before carbon tiles are installed on the wall protection elements will help to identify critical locations and to avoid such discharge conditions in later higher-power operation. 


\section{Conclusions}

In OP1.1, W7-X will be limited to a low heating energy input per discharge, not all heating systems will be available, the diagnostics will be under commissioning and may not yet be routinely available, and their number will be limited. Nevertheless, there will be the potential to perform several interesting and important investigations in this first campaign of plasma operation. Apart from the integrated commissioning of the control systems and the test of the diagnostics, the high priority physics goals will be the assessment of the magnetic field quality by flux surface mapping, the investigation of the plasma breakdown behaviour in helium and hydrogen, and some aspects of edge transport in a helical limiter plasma for later comparison with divertor plasmas. In addition, there will be the opportunity for basic tests of electron cyclotron current drive and for first measurements of plasma turbulence and transport.

\section{Acknowledgements}

We thank C. Beidler, A. Dinklage, P. Helander and H. Maaßberg for their useful feedback on this paper. This work has been carried out within the framework of the EUROfusion Consortium and has received funding from the Euratom research and training programme 2014-2018 under grant agreement no. 633053. The views and opinions expressed herein do not necessarily reflect those of the European Commission.

\section{References}

[1] C. Beidler et al., Fusion Technology 17, 148 (1990).

[2] H.-S. Bosch et al., Nucl. Fusion 53, 126001 (2013).

[3] H.-S. Bosch et al., IEEE Trans. Plasma Sci. 42, 432 (2014).

[4] B. Mendelevitch et al., Fusion Eng. Design 88, 1660 (2013), Proceedings of the 27th Symposium on Fusion Technology (SOFT-27), Liège, Belgium, September 24-28, 2012.

[5] S. A. Bozhenkov et al., Limiter for the early operation phase of W7-X, in 41st EPS Conference on Plasma Physics, Berlin, Germany, 23-27 June 2014, edited by S. Ratynskaia, P. Mantica, A. Benuzzi-Mounaix, G. Dilecce, and R. Bingham, volume 38F, European Physical Society, 2014, paper P-1.080, http://ocs.ciemat.es/ EPS2014PAP/pdf/P1.080.pdf.

[6] A. Peacock et al., Fusion Eng. Design 84, 1475 (2009), Proceedings of the 25th Symposium on Fusion Technology (SOFT-25), Rostock, Germany, 15-19 September 2008.

[7] G. Grieger et al., Phys. Fluids B 4, 2081 (1992). 
[8] J. Boscary et al., Fusion Eng. Design 86, 572 (2011), Proceedings of the 26th Symposium on Fusion Technology (SOFT-26), Porto, Portugal, September 27-October 1, 2010.

[9] J. Kisslinger, C. Beidler, E. Harmeyer, F. Rau, and H. Wobig, Magnetic field and coil systems of the modular helias configuration HS 5-10, in Fusion Technology 1990, edited by B. E. Keen, M. Huguet, and R. Hemsworth, pages 1520-1524, Elsevier Science Publishers B. V., 1991, Proceedings of the 16th Symposium on Fusion Technology, London, U. K., 3-7 September 1990.

[10] V. Erckmann et al., Fusion Sci. Tech. 52, 291 (2007).

[11] H. Grote et al., J. Nucl. Mater. 313-316, 1298 (2003), Proceedings of Plasma-Surface Interactions in Controlled Fusion Devices 15, Gifu, Japan, 26-31 May 2002.

[12] R. König et al., The set of diagnostics for the first operation campaign of the Wendelstein 7-X stellarator, in 1st EPS Conference on Plasma Diagnostics (ECPD, Villa Mondragone, Frascati, Italy, 14-17 April 2015), 2015, accepted for publication in JINST.

[13] B. Wiegel et al., Radiation Protection Dosimetry 161, 326 (2014), Proceedings of the Twelfth International Symposium on Neutron and Ion Dosimetry (Neudos 12), 3-7 June 2013, Aix-en-Provence, France.

[14] G. Kocsis et al., Fusion Eng. Design 96-97, 808 (2015), Proceedings of the 28th Symposium On Fusion Technology (SOFT-28), San Sebástian, Spain, 29 September3 October 2014.

[15] M. Preynas et al., A near infra-red video system as a protective diagnostic for ECRH operation in the Wendelstein 7-X stellarator, 2015, submitted to Rev. Sci. Instrum.

[16] G. Haas and H.-S. Bosch, Vacuum 51, 39 (1998).

[17] M. Hirsch et al., Proc. of Science (ECPD2015), 111 (2015), Proceedings of the 1st EPS conference on Plasma Diagnostics, 14-17 April 2015, Frascati, Italy.

[18] J. Cantarini, J. P. Knauer, and E. Pasch: "Design and Study of the Observation Optics for the Thomson Scattering Planned at Wendelstein 7-X" in AIP Conf. Proc. 993 (2008), H.-J. Hartfuss, M. Dudeck, J. Musielok, and M. J. Sadowski, editors, pages 191-194, Proceedings of "PLASMA 2007", 16-19 October 2007, Greifswald, Germany.

[19] D. Zhang et al., Rev. Sci. Instrum. 81, 10E134 (2010), Proceedings of the 18th Topical Conference on High-Temperature Plasma Diagnostics, Wildwood, New Jersey, 16-20 May 2010.

[20] M. Endler et al., Fusion Eng. Design 100, 468 (2015). 
[21] G. Bertschinger, W. Biel, H. Jaegers, and O. Marchuk, Rev. Sci. Instrum. 75, 3727 (2004), Proceedings of the 15th Topical Conference on High-Temperature Plasma Diagnostics, San Diego, CA, 2004.

[22] A. Langenberg et al., Forward modeling of a high resolution X-ray imaging crystal spectrometer for the Wendelstein 7-X stellarator, in 41st EPS Conference on Plasma Physics, Berlin, Germany, 23-27 June 2014, edited by S. Ratynskaia, P. Mantica, A. Benuzzi-Mounaix, G. Dilecce, and R. Bingham, volume 38F, European Physical Society, 2014, paper P-1.074, http://ocs.ciemat.es/EPS2014PAP/pdf/P1.074.pdf.

[23] N. A. Pablant et al., Measurement of core plasma temperature and rotation on W7-X made available by the x-ray imaging crystal spectrometer (XICS), in 41st EPS Conference on Plasma Physics, Berlin, Germany, 23-27 June 2014, edited by S. Ratynskaia, P. Mantica, A. Benuzzi-Mounaix, G. Dilecce, and R. Bingham, volume 38F, European Physical Society, 2014, paper P-1.076, http://ocs.ciemat.es/ EPS2014PAP/pdf/P1.076.pdf.

[24] M. Kubkowska et al., Laboratory tests of the pulse height analysis system for Wendelstein 7-X, in 1st EPS Conference on Plasma Diagnostics (ECPD, Villa Mondragone, Frascati, Italy, 14-17 April 2015), 2015, to be published in JINST, preprint available at http://www.euro-fusionscipub.org/wp-content/uploads/2015/ 08/WPS1PR1504.pdf.

[25] W. Biel, A. Greiche, R. Burhenn, E. Jourdain, and D. Lepere, Rev. Sci. Instrum. 77, 10F305 (2006), Proceedings of the 16th Topical Conference on High-Temperature Plasma Diagnostics, Williamsburg, VA, USA, 7-11 May 2006.

[26] A. Greiche, W. Biel, O. Marchuk, and R. Burhenn, Rev. Sci. Instrum. 79, 093504 (2008).

[27] O. Neubauer et al., Fusion Eng. Design 96-97, 891 (2015), Proceedings of the 28th Symposium On Fusion Technology (SOFT-28), San Sebástian, Spain, 29 September3 October 2014.

[28] T. Windisch et al., Reflectometry fluctuation diagnostics at Wendelstein 7-X, in 42nd EPS Conference on Plasma Physics, Lisbon, Portugal, 22-26 June 2015, edited by R. Bingham, W. Suttrop, S. Atzeni, R. Foest, and K. McClements, volume 39E, European Physical Society, 2015, paper P-1.163, http://ocs.ciemat.es/EPS2014PAP/ pdf/P1.163.pdf.

[29] R. Jaenicke et al., Nucl. Fusion 33, 687 (1993).

[30] T. Sunn Pedersen et al., Phys. Plasmas 13, 012502 (2006).

[31] M. Otte et al.: "The WEGA Stellarator: Results and Prospects" in AIP Conf. Proc. 993 (2008), H.-J. Hartfuss, M. Dudeck, J. Musielok, and M. J. Sadowski, editors, pages 3-10, Proceedings of "PLASMA 2007", 16-19 October 2007, Greifswald, Germany. 
[32] J. Kißlinger and T. Andreeva, Fusion Eng. Design 74, 623 (2005), Proceedings of the 23rd Symposium on Fusion Technology, Venice, Italy, 20-24 September 2004.

[33] K. Risse, F. Füllenbach, T. Rummel, M. Mardenfeld, and X. Zhao, IEEE Trans. Plasma Sci. 42, 449 (2014).

[34] S. A. Lazerson et al., Error field and magnetic diagnostic modeling for W7-X, in 41st EPS Conference on Plasma Physics, Berlin, Germany, 23-27 June 2014, edited by S. Ratynskaia, P. Mantica, A. Benuzzi-Mounaix, G. Dilecce, and R. Bingham, volume 38F, European Physical Society, 2014, paper P-1.073, http://ocs.ciemat.es/ EPS2014PAP/pdf/P1.073.pdf.

[35] M. Preynas et al., Study of plasma start-up initiated by second harmonic electron cyclotron resonance heating on WEGA experiment, in AIP Conf. Proc., volume 1580, pages 498-501, American Institute of Physics, 2014, Proceedings of "Radiofrequency Power in Plasmas".

[36] W. I. van Rij and S. P. Hirshman, Phys. Fluids B 1, 563 (1989).

[37] Y. Turkin et al., Phys. Plasmas 18, 022505 (2011).

[38] N. B. Marushchenko, Y. Turkin, and H. Maassberg, Comp. Phys. Comm. 185, 165 (2014).

[39] H. Yamada et al., Nucl. Fusion 45, 1684 (2005).

[40] Y. Feng et al., Neutral transport behavior expected for the first limiter plasmas in W7-X, in 41st EPS Conference on Plasma Physics, Berlin, Germany, 23-27 June 2014, edited by S. Ratynskaia, P. Mantica, A. Benuzzi-Mounaix, G. Dilecce, and R. Bingham, volume 38F, European Physical Society, 2014, paper P-1.079, http:// ocs.ciemat.es/EPS2014PAP/pdf/P1.079.pdf.

[41] F. Effenberg et al., Investigation of impurity radiation in Wendelstein 7-X startup plasmas with EMC3-Eirene, in 56th Annual Meeting of the APS Division of Plasma Physics, New Orleans, LA, 2014.

[42] J. Kißlinger et al., Island divertor for the stellarator Wendelstein 7-X, in 21st EPS Conference on Controlled Fusion and Plasma Physics (Montpellier), edited by E. Joffrin, P. Platz, and P. E. Stott, volume 18B, part I, pages 368-371, European Physical Society, 1994.

[43] R. J. Goldston, Nucl. Fusion 52, 013009 (2012).

[44] F. Sardei et al., J. Nucl. Mater. 241-243, 135 (1997), Proceedings of the Twelveth International Conference on Plasma-Surface Interactions in Controlled Fusion Devices, Saint-Raphaël, France, 20-24 May 1996.

[45] M. Kick et al., Plasma Phys. Controlled Fusion 41, A549 (1999). 